\title{
Frequency blue shift of terahertz radiation from femtosecond laser induced air plasmas
}

\author{
Zhen Zhang, ${ }^{1,5} \cdot$ Yanping Chen, ${ }^{1,2,{ }^{*}} \cdot$ Zhelin Zhang, $^{1,2,4} \cdot$ Tianhao Xia, $^{1,2} \cdot$ Jiayang Zhang, $^{1,2} \cdot$ Zhengming $^{2}$ \\ Sheng, $1,2,3,4$ \\ ${ }^{1}$ Key Laboratory for Laser Plasma (Ministry of Education), School of Physics and Astronomy, Shanghai Jiao Tong University, Shanghai 200240, \\ China \\ 2 IFSA Collaborative Innovation Center, Shanghai Jiao Tong University, Shanghai 200240, China \\ ${ }^{3}$ Department of Physics, SUPA, University of Strathclyde, Glasgow G4 ONG, UK \\ ${ }^{4}$ Tsung-Dao Lee Institute, Shanghai Jiao Tong University, Shanghai 200240, China \\ ${ }^{5}$ Laboratory of Quantum Information Technology, Chongqing University of Posts and Telecommunications, Chongqing 400065, China \\ *Corresponding author: yanping.chen@sjtu.edu.cn
}

\begin{abstract}
The peak frequency of the terahertz radiation generated from the femtosecond laser induced air plasmas is blue shifted with the increased second harmonics intensity when a high voltage is added upon the air plasmas. This blue shift is the result of the DC-biased field from the high voltage plus the AC-biased field from the second harmonics, indicating that this terahertz radiation frequency peak is tunable by varying the two field components. This tunable frequency peak is significant to various applications such as remote sensing and material characterization.
\end{abstract}

\section{Introduction}

Broadband terahertz $(\mathrm{THz})$ radiation provides a unique tool in a wide range of applications from material characterization and chemical sensing/identification to label-free genetic analysis [1, 2]. The generation and detection techniques of broadband $\mathrm{THz}$ radiation have been widely studied and investigated in the last few decades [3]. The flexibility of application of terahertz radiation can be expanded by manipulating the terahertz radiation in different aspects such as the output power tuning [4-9], spectrum tuning [10-15], polarization tuning [16-18] and so on. Among these, the frequency tuning technique is still highly desirable and challenging.

Generally, there are two different ways for terahertz frequency tuning, one is to use a spatial light modulator (SLM) to change the spot distribution of the pump laser [10,11], the other is based on difference frequency mixing in nonlinear crystals [12-15]. The peak frequency can be tuned from $2 \mathrm{THz}$ to $4.5 \mathrm{THz}$ at a relatively low laser energy (about $1 \mathrm{~mJ} /$ pulse) using a SLM [10]. The method of difference frequency mixing in the nonlinear crystal is mainly suitable for tuning the high-frequency terahertz wave with high efficiency. The carrier-envelop phase-locked laser and a near-degenerate optical parametric amplifier are needed to tune the frequency from $15 \mathrm{THz}$ to $37.5 \mathrm{THz}$ [12]. However, both these two methods are limited by the damage thresholds of either the SLM or the nonlinear crystals. Besides, the latter also requires complicated experimental configurations.

The terahertz frequency shift can also be obtained by manipulating femtosecond laser induced air filaments. Mitryukovskiy et al [19] proposed a method for $\mathrm{THz}$ radiation with its peak frequency tunable from $0.1 \mathrm{THz}$ to $1 \mathrm{THz}$ by simulating $\mathrm{THz}$ radiation from two filaments in a Vee arrangement. In order to select a desired frequency, one needs to control the temporal separation as well as the angle between two filaments simultaneously. So, this method also requires a complicated experimental configuration and need more effort to align the experimental setup. With two-color laser induced air filaments, Wang et al. [20] found that the frequency of the terahertz radiation has a certain redshift tendency when applying a DC-biased field whose direction is parallel to the polarization of the $800 \mathrm{~nm}$ laser.

In this article, we provide a simple method for peak frequency shift of broadband terahertz radiation based on laser-produced air plasmas by applying AC-biased fields (the second harmonic of the incident laser) and DC-biased fields simultaneously. The focus of our research is concentrated on the effect of the rotation 
angle of the BBO crystal on the frequency shift of the terahertz radiation. Experimental results show the tunability of the terahertz frequency by changing the intensity of the second harmonics via the rotation of the BBO crystal angle and by applying the DC-biased field. The physical mechanism of such frequency tuning will then be revealed. This work provides a simple way to tune the terahertz spectrum and supplements the spectral tuning range of the terahertz waves.

\section{Experimental setup}

The experimental setup is illustrated in Fig. 1. An $800 \mathrm{~nm}, 40 \mathrm{fs}$ laser pulse was focused by a plano-convex lens of $30 \mathrm{~cm}$ focal length to form a filament in the air. The focused pump pulse passes through a $0.2-\mathrm{mm}$-thick type-I BBO crystal to produce the second harmonic wave. The $\mathrm{BBO}$ rotation angle $\theta$ was changed from 0 to 90 degrees. A DC-biased field of $8 \mathrm{kV} / \mathrm{cm}$ was applied perpendicularly to the laser filament. The terahertz pulses generated from the laser filament were collected in the forward direction through a pair of parabolic mirrors and detected by electro-optic sampling (EOS) technique with a $<110>\mathrm{ZnTe}$ crystal of $1.5 \mathrm{~mm}$ thickness.

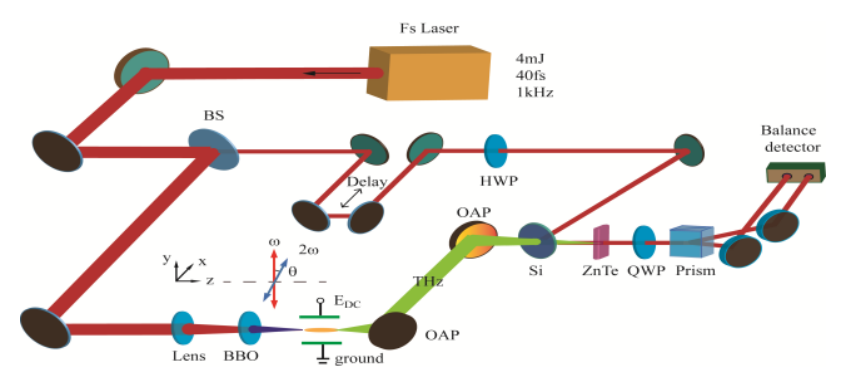

Fig. 1. (Color online) Schematic diagram of the experimental setup. A fundamental laser pulse propagates through a BBO crystal, generating a second harmonic pulse. The polarization of the laser pulse is in the vertical direction. The plasma filament is generated by the two-color laser pulse in the focal region of the lens, and a high voltage of $8 \mathrm{kV}$ is applied over the filament along the same direction as the polarization of the fundamental laser. Where $\theta$ refers to the angle of the BBO crystal. THz emission from the filament is detected via electro-optic sampling technique after being collected by a pair of off-axis parabolic mirrors. BS: beam splitter; OAP: off-axis parabolic; HWP: half-wave plate; QWP: quarter wave plate.

\section{Frequency tuning and its mechanism}

We adjust the peak frequency of the terahertz radiation from the DC-biased two-color air plasmas by rotating the angle of the
BBO crystal $\theta$, as shown in Fig. 2(a) and Fig. 2(b). When $\theta=90^{\circ}$, i.e., the polarization of the fundamental wave is perpendicular to the $e$-axis of the $\mathrm{BBO}$ crystal, the conversion efficiency of the second harmonic is optimized. The peak frequency of spectrum shifts from $0.46 \mathrm{THz}$ to $0.70 \mathrm{THz}$ with the angle $\theta$ changing from $0^{\circ}$ to $45^{\circ}$. When $\theta$ is over $45^{\circ}$, the peak frequency of the terahertz spectrum remains substantially at $0.70 \mathrm{THz}$.

This frequency change might be caused either by AC-biased fields or by DC-biased fields. To interpret this experimental observation, Fig. 2(c) shows the waveforms of terahertz radiation from two-color air plasmas without the DC-biased fields. The corresponding spectra from the Fourier transform are shown in Fig. 2(d). We find that the peak frequencies of the terahertz spectra in Fig. 2(d) remain roughly unchanged (about $0.70 \mathrm{THz}$, marked as a dashed line in the figure) when the angle $\theta$ increases from $0^{\circ}$ to $90^{\circ}$. Figure 2(e) represents the terahertz waves contributed by the DC-biased air plasmas, which is obtained by subtracting the waveforms of (c) from those of (a) [21]. The corresponding Fourier transformed spectra are shown in Fig. 2(f). Both their peak frequencies (about $0.46 \mathrm{THz}$, marked as the dashed line) and intensities do not change with the angle $\theta$. It looks like in Fig 2(f) the slightly shifts is smaller than the frequency changes in Fig. 2(b). Consequently, neither the AC-biased fields nor the DC-biased fields causes the blue shift of the terahertz peak frequency as the angle $\theta$ increases. This frequency tuning phenomenon occurs only when both the AC-biased fields and the DC-biased fields are applied on the air plasma.

When the angle $\theta$ is $0^{\circ}$, i.e., the polarization direction of the $800 \mathrm{~nm}$ laser is parallel to the e-axis of the BBO crystal, the conversion efficiency of the second harmonic is zero. In this case, the single-color pulse ionizes the air and generates plasmas. The $\mathrm{THz}$ radiation is mainly contributed by the DC-biased air plasmas, resulting in a peak frequency of about $0.46 \mathrm{THz}$. When the angle $\theta$ increases, the conversion efficiency as well as the intensity of the second harmonic increases. In this case, the THz radiation is contributed by AC-biased air plasma appears with a fixed peak frequency of $0.70 \mathrm{THz}$ [Fig. 2(d)]. Its intensity grows with the increase of the second harmonic. Therefore, the measured terahertz radiation at far field is contributed by two mechanisms. One is the ionized photocurrent driven by the DC-biased electric field, and another is driven by the photocurrent induced by the asymmetric laser field inside the AC-biased air plasma (Details in Section 4). As a result, the peak frequency of the observed terahertz radiation is dominated by the combined effect of AC-bias and DC-bias. As the angle $\theta$ increases from $0^{\circ}$ to $45^{\circ}$, the 
peak frequency of the $\mathrm{THz}$ radiation changes from $0.46 \mathrm{THz}$ to $0.70 \mathrm{THz}$ because the radiation contributed by AC-bias becomes more and more significant. When the angle $\theta$ exceeds $45^{\circ}$, the intensity of terahertz waves contributed by AC-bias is much higher than those contributed by DC-bias [Fig. 2(c) and 2(e)]. Thus, the peak frequency of observed terahertz radiation is fixed at $0.70 \mathrm{THz}$, dominated by $\mathrm{AC}$-biased terahertz radiation.
In the following, we discuss the relationship between the $\mathrm{THz}$ peak frequencies and their generation mechanisms. Generally, $\mathrm{THz}$ radiation from laser plasmas is due to electron oscillation inside the plasma region. Its frequency is equivalent to the plasma frequency [8].
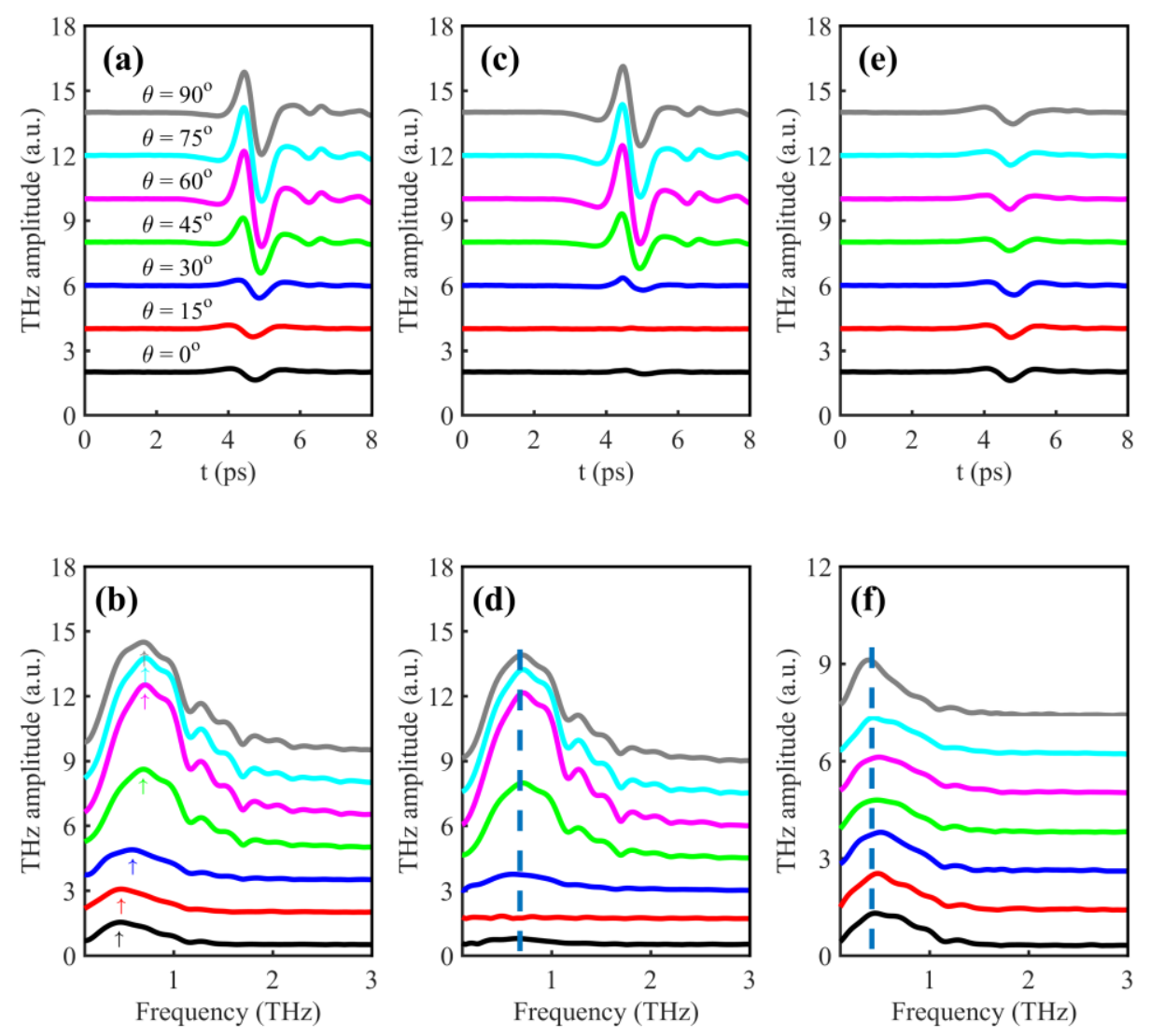

Fig. 2. The THz waveforms and their corresponding spectra from the DC-biased two-color air plasmas (a and b), the two-color air plasmas only (c and d), and the DC-biased air plasmas only (e and f). The laser energy is $1 \mathrm{~mJ}$.

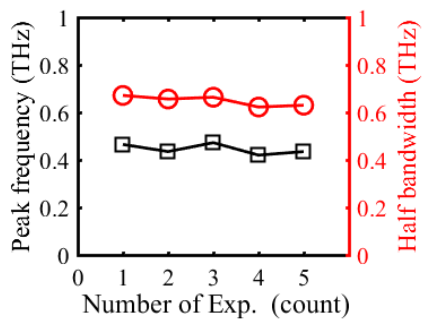

Fig. 3. Frequency repeatability of the peak frequency of the frequency spectrum and half-width of the frequency spectrum. The laser energy is $1.0 \mathrm{~mJ}$.
Due to the frequency repeatability from the experiment is important for peak frequency analysis, we have made an attempt to estimate experimental uncertainties of the frequency spectra. Fig. 3 shows the peak frequency of the frequency spectrum and the full width at half maximum of the frequency spectrum for different experimental counts when the laser energy is $1.0 \mathrm{~mJ}$. As the number of experiments changes, the peak frequency of the frequency spectrum and the bandwidth of the spectrum remain basically the same. We found that the maximum error of the peak frequency of the frequency spectrum is $0.03 \mathrm{THz}$ and the maximum error of the half-height of the frequency spectrum is $0.03 \mathrm{THz}$. We have found that the frequency shift is greater than 
that which might be perceived by random chance (i.e. the noise and jitter in the laser pulses and $\mathrm{THz}$ detector).

Inside the plasma region, the electron density as well as the plasma frequency is relatively higher in the inner part of the plasma column and relatively lower in the outer part [21]. It is well-known that electromagnetic waves will be obstructed while penetrating into high density plasmas, where there is a penetration depth given by $\delta=c\left(\omega_{p e}^{2}-\omega^{2}\right)^{-1 / 2}$, where $\omega$ is the frequency of the electromagnetic wave and $\omega_{p e}=\left(4 \pi n_{0} e^{2} / m_{e}\right)^{1 / 2}$ is the electron plasma frequency [22]. Where $n_{0}$ is the electron density and $m_{e}$ is the mass of the electron. As the DC-bias field is applied, a sheath field will be built up more quickly in the inner layer. As a result, only electrons in the outer layer of the plasma column will be accelerated to form a transient current for $\mathrm{THz}$ radiation. As a result, the $\mathrm{THz}$ radiation contributed by the DC-biased field mainly comes from the outer layer, with a lower peak frequency. When the second harmonic is applied on the plasma, the ionization current is mainly formed in the inner layer of the plasma column. This means that the $\mathrm{THz}$ radiation driven by the AC-biased field is generated from there with a relatively higher peak frequency.

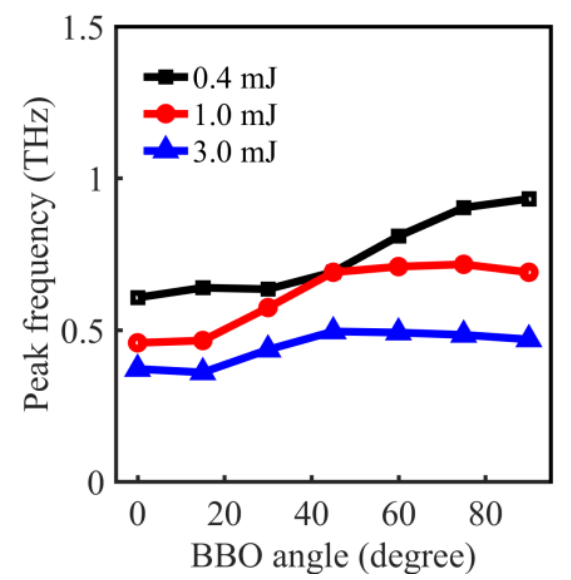

Fig. 4. The peak frequencies of the terahertz radiation from the DC-biased two-color laser plasma as a function of the angle $\theta$.

Similar frequency tuning phenomena are found in our experiment when the incident laser energy is changed between $0.4 \mathrm{~mJ}$ and $3 \mathrm{~mJ}$, as shown in Fig. 4. The tuning range of the THz peak frequency is from $0.60 \mathrm{THz}$ to $0.93 \mathrm{THz}$ at $0.4 \mathrm{~mJ}$ laser energy, while the tuning range is $0.37 \mathrm{THz}$ to $0.49 \mathrm{THz}$ for $3 \mathrm{~mJ}$ laser energy. Note that as the laser energy decreases, the tuning range of the $\mathrm{THz}$ peak frequency is expanded from $0.12 \mathrm{THz}$ to $0.33 \mathrm{THz}$. With the increase of laser energy, the overall spectrum is shifted to a lower frequency at all BBO angles. This can be explained by combined effect of two processes during filamentation [23]. First, a laser induced plasma zone is extended on the propagation axis when laser energy increases. The plasma density is relatively lower in the extended plasma zone than that at the geometric focus because convergence effect of Kerr focusing (extended plasma zone) is weaker than a converging lens (geometric focus). Thus, the averaged plasma density in the plasma column gets lower with increased laser energy. The second is the decrease in the temporal and spatial overlap of the fundamental and second harmonic pulses. The length of the filament increases with the laser energy, resulting in the decrease of the temporal overlap between the fundamental wave and its second harmonics [24] due to the presence of the moving focus [25] and the pulse self-compression effect [26]. This leads to a decrease in the ionization rate of the two-color fields ionized air, thus a decrease in the electron density in the plasma.

\section{Conclusions}

In summary, we demonstrate a method for frequency shift of broadband $\mathrm{THz}$ radiations from femtosecond laser ionized air plasmas. With the two-color laser pulses and the external DC-bias field applied on the air plasma, the relationship between the peak frequency of $\mathrm{THz}$ waves and the rotation angle of the $\mathrm{BBO}$ crystal is investigated. Due to the combined processes in the $\mathrm{THz}$ radiation generation by the applied DC electric field and by the two-color laser electric fields induced air filaments, a shift of the $\mathrm{THz}$ peak frequency is realized. The tunability of the $\mathrm{THz}$ spectrum is important for understanding the mechanisms of $\mathrm{THz}$ wave generation and is expected to be applied to frequency manipulation of $\mathrm{THz}$ radiation.

Funding. The National Natural Science Foundation of China (Grant No. 11774228, 12074250, 11655002, 11721091, 11991070) and Science and Technology Commission of Shanghai Municipality (Grant No. 16DZ2260200).

Disclosures: The authors declare no conflicts of interest.

\section{References}

1. M. Brucherseifer, M. Nagel, P. Haring Bolivar, H. Kurz, A. Bosserhoff, and R. Büttner, Appl. Phys. Lett. 77, 4049-4051 (2000).

2. D. Mittleman, M. Gupta, R. Neelamani, R. Baraniuk, J. Rudd, and M. Koch, Appl. Phys. B. 68, 1085-1094 (1999). 
3. M. Thomson, M. Kreß, T. Löffler, and H. Roskos, Laser \& Photonics Review. 1, 349-368 (2007).

4. J. Zhao, Y. Zhang, Z. Wang, W. Chu, B. Zeng, W. Liu, Y. Cheng, and Z. Xu, Laser Phys. Lett. 11, 095302 (2014).

5. N. Panov, V. Andreeva, O. Kosareva, A. Shkurinov, V. Makarov, L. Bergé, and S. Chin, Laser Phys. Lett. 11, 125401 (2014).

6. D. Cook and R. Hochstrasser, Opt. Lett. 25, 1210-1212 (2000).

7. S. Y. Stremoukhov and A. V. Andreev, Laser Phys. Lett. 12, 015402 (2014).

8. A. Houard, Y. Liu, B. Prade, V. T. Tikhonchuk, and A. Mysyrowicz, Phys. Rev. Lett. 100, 255006 (2008).

9. T.-J. Wang, C. Marceau, S. Yuan, Y. Chen, Q. Wang, F. Théberge, M. Châteauneuf, J. Dubois, and S. Chin, Laser Phys. Lett. 8, 57 (2010).

10. M. Yamaguchi and J. Das, JOSA B 26, A90-A94 (2009).

11. J. Das and M. Yamaguchi, Opt. Express 18, 7038-7046 (2010).

12. T. Balčiūnas, D. Lorenc, M. Ivanov, O. Smirnova, A. Zheltikov, D. Dietze, K. Unterrainer, T. Rathje, G Paulus, and A. Baltuška, Opt. Express 23, 15278-15289 (2015).

13. T. Tanabe, K. Suto, J.-i. Nishizawa, K. Saito, and T. Kimura, Appl. Phys. Lett 83, 237-239 (2003).

14. K. Vodopyanov, M. Fejer, X. Yu, J. Harris, Y.-S. Lee, W. C. Hurlbut, V. Kozlov, D. Bliss, and C. Lynch, Applied Physics Letters 89, 141119 (2006).

15. K. L. Vodopyanov, Laser \& Photonics Reviews 2, 11-25 (2008).

16. J. Dai, N. Karpowicz, and X.-C. Zhang, Physical Review Letters 103, 023001 (2009).

17. H. Wen and A. M. Lindenberg, Physical Review Letters 103, 023902 (2009).

18. Z. Zhang, Y. Chen, S. Cui, F. He, M. Chen, Z. Zhang, J. Yu, L. Chen, Z. Sheng, and J. Zhang, Nature Photonics 12, 554 (2018).

19. S. Mitryukovskiy, Y. Liu, B. Prade, A. Houard and A. Mysyrowicz, Laser Physics 24, 094009 (2014).

20. T.-J. Wang, C. Marceau, Y. Chen, S. Yuan, F. Théberge, M. Châteauneuf, J. Dubois, and S. L. Chin, Applied Physics Letters 96, 211113 (2010).

21. J. Zhao, W. Chu, L. Guo, Z. Wang, J. Yang, W. Liu, Y. Cheng, and Z. Xu, Scientific Reports 4, 3880(2014).

22. Y. Chen, T.-j. Wang, C. Marceau, F. Théberge, M. Châteauneuf, J. Dubois, O. Kosareva, and S. L. Chin, Applied Physics Letters 95, 101101 (2009).

23. F. Théberge, W. Liu, P. T. Simard, A. Becker, and S. L. Chin, Physical Review E 74, 036406 (2006).

24. Z. Zhang, N. Panov, V. Andreeva, Z. Zhang, A. Slepkov, D. Shipilo, M. Thomson, T.-J. Wang, I. Babushkin, and A. Demircan, Applied Physics Letters 113, 241103 (2018).

25. A. Brodeur, C. Chien, F. Ilkov, S. Chin, O. Kosareva, and V. Kandidov, Optics Letters 22, 304-306 (1997).

26. G. Stibenz, N. Zhavoronkov, and G. Steinmeyer, Optics Letters 31, 274-276 (2006). 